Published in:Journal of the Acoustical Society of America, 100(2), pt. 1, August 1996, pp. 933-944 (C) (1996) Acoustical Society of America. This article may be downloaded for personal use only. doi:10.1121/1.416252 http://scitation.aip.org/content/asa/journal/jasa/100/2/10.1121/1.416252

\title{
On the incorporation of moiré shape measurements in finite- element models of the cat eardrum
}

\author{
W. Robert J. Funnella) \\ Departments of BioMedical Engineering \& Otolaryngology, McGill University, 3775, rue University, \\ Montréal, Québec H3A 2B4, Canada \\ Willem F. Decraemer ${ }^{\text {b) }}$ \\ Department of Biomedical Physics, University of Antwerp (RUCA), Groenenborgerlaan 171, \\ Antwerpen B-2020, Belgium
}

(Received 15 March 1995; accepted for publication 11 December 1995)

\begin{abstract}
The mechanical behavior of the eardrum has previously been shown to depend critically on its shape, but accurate shape measurements have been difficult to make. Phase-shift moiré topography provides a valuable technique for measuring such shapes, and measurement in the presence of large static pressures facilitates the determination of the boundaries of the pars tensa, pars flaccida, and manubrium. New measurements of the shape of the cat eardrum are presented. The presence of hysteresis in the pressure-displacement response is demonstrated. The shapes are incorporated in individualized finite-element models for four different ears, and the variability between and within animals is examined. Fixed-manubrium low-frequency displacements are simulated and compared for the different models. (C) 1996 Acoustical Society of America.
\end{abstract}

PACS numbers: 43.64.Bt, 43.64.Ha [RAS]

\section{INTRODUCTION}

The mechanical behavior of the eardrum critically depends on its shape, but until recently the available shape data (Helmholtz, 1869; Kojo, 1954; Kirikae, 1960) were neither precise nor detailed. As a consequence, the shape was approximated in our finite-element models by only two parameters: the depth of the cone, and an average or typical radius of curvature for the sides of the cone (Funnell and Laszlo, 1978).

Moiré topography is an optical technique involving the projection of a grating of parallel lines onto the surface being measured, and offers a convenient noncontacting method for measuring the shapes of small objects. In the first attempts to measure eardrum shape using moiré topography (Khanna and Tonndorf, $1975 \mathrm{a}, \mathrm{b})$ the measurements were made on Silastic castings in order to obtain adequate optical contrast. This caused the boundary definition to be poor because the castings could not accurately replicate the very narrow space between the ear-canal wall and parts of the eardrum. Furthermore, the moiré technique that was used resulted in fringes which had to be counted to determine $z$ coordinates, which made the analysis very difficult and provided only limited depth resolution (Funnell, 1981). The analysis is greatly simplified by the use of the phase-shift moiré technique, which involves the combination of four moiré fringe images, resulting in images in which the value of each pixel is directly related to the $z$ coordinate (Dirckx et al., 1988; Dirckx and Decraemer, 1989). The technique also provides greatly improved depth resolution, with an accuracy of $20 \mu \mathrm{m}$ (Dirckx and Decraemer, 1990).

It also became possible to measure the eardrum directly

\footnotetext{
${ }^{a)}$ Electronic mail: R.Funnell@med.mcgill.ca

${ }^{b)}$ Electronic mail: Wimdec@ruca.ua.ac.be
}

rather than with castings, and to measure the shape under the influence of sequences of static pressures applied in the middle-ear air cavities (Decraemer and Dirckx, 1991). Elimination of the castings removed one source of error in defining the boundary of the eardrum, but in some regions there is no distinct landmark to demarcate the boundary. Application of static pressures facilitates identification of the boundary in such regions because the eardrum moves in response to the pressures while the ear-canal tissue does not. In other regions, however, the boundary is hidden under overhanging tissue which cannot be removed without damaging the eardrum. In these cases a different approach is required, as described below.

Unpressurized eardrum shapes measured with the phaseshift moiré technique have previously been presented for human (Decraemer et al., 1991) and cat (Decraemer and Dirckx, 1991). Shape data with static pressures applied have previously been presented for the human eardrum (Dirckx and Decraemer, 1991, 1992) and for a single cat (Decraemer and Dirckx, 1991; Funnell et al., 1993).

In this paper the moiré shape-measurement technique as applied to the cat eardrum is briefly summarized in Sec. I, and the procedures used to analyze the shapes and to incorporate them in finite-element models are described in Sec. II. New shape data for the cat eardrum are then presented in Sec. III and simulation results for finite-element models incorporating the shape data are presented in Sec. IV. The simulations include some that use a parametric representation of eardrum shape similar to that used in previous models.

\section{EXPERIMENTAL SHAPE MEASUREMENT}

The experimental technique has been described previously (Decraemer et al., 1991; Dirckx and Decraemer, 
1991). The fresh temporal bones to be measured are obtained from cats that have been used for purposes unrelated to the auditory system. The cats are sacrificed with an intracardiac injection of pentobarbital $\mathrm{Na}$ solution $(60 \mathrm{mg} / \mathrm{kg}$ bodyweight). The dissection of the temporal bone starts about 15 min post mortem. In order to provide a good view of the eardrum, the ear canal is resected to within about $0.5 \mathrm{~mm}$ of the tympanic ring, as close as possible without damaging the eardrum. Static pressures of as much as $\pm 220 \mathrm{~mm} \mathrm{H}_{2} \mathrm{O}$ are applied to the middle ear through a small hole drilled in the bulla wall. So that the eardrum does not dry out, it is regularly moistened during the preparation, but not during the recording time itself which starts about $6 \mathrm{~h}$ post mortem. Drying out of the eardrum is fairly slow because the epithelial layer on the lateral side of the eardrum is relatively thick and is covered by a layer of Chinese ink (which is water based); and because the middle-ear air cavity is kept closed, protecting the very thin mucosal layer on the medial side of the drum. No shape changes were observed when the air in the ear canal was humidified by breathing into it.

Moiré interferograms of the eardrum are obtained by casting the shadow of a grating of parallel lines onto a temporal bone placed close behind the grating. The shadow is a set of deformed lines and forms a moiré interference pattern when observed through the line grating. The moire images are recorded using a CCD camera and a frame store. For the optical setup used for the present work, the scaling of the moiré images was $45.8 \mu \mathrm{m} /$ pixel horizontally and $31.7 \mu \mathrm{m} /$ pixel vertically. During the recording of each image the grating is moved in its plane in order to average out the grating lines. Four phase-shifted images are obtained by moving the object slightly along the axis perpendicular to the grating between images. The four images are then combined pixel by pixel to form a single image in which the value of each pixel specifies the $z$ coordinate of a point (Dirckx et al., 1988). The computation of $z$ at each pixel involves an arctangent and the result is therefore "wrapped" into the range 0 to $2 \pi k$, where $k$ is a calibration constant $(\mathrm{m} / \mathrm{rad})$. The $z$ coordinates must be "unwrapped" by adding multiples of $2 \pi k$ where required. For the measurements reported here, the grating had 4 lines $/ \mathrm{mm}$, resulting in a $z$ calibration of 118 $\mu \mathrm{m} / \mathrm{rad}$. This corresponds to $0.74 \mathrm{~mm}$ for $2 \pi \mathrm{rad}$, which is the size of the ambiguities that must be resolved by unwrapping as discussed below.

The moiré technique requires a diffusely reflecting surface in order to obtain good optical contrast, but the eardrum reflects only poorly, so something must be done to make it more reflective. Application of white Chinese ink (Pelican Drawing Ink A, 18 White) has been shown to result in a layer which is only about $10 \mu \mathrm{m}$ thick and quite uniform (Decraemer and Dirckx, 1991; Stoffels, 1993). The measured eardrum surfaces were generally quite smooth, except in one animal in which there were artefacts in two locations near the manubrium. The weight of the ink is equivalent (assuming a density of twice that of water) to a pressure on the order of only $20 \mu \mathrm{m} \mathrm{H}_{2} \mathrm{O}$, so its effect on the shape of the eardrum is negligible.

In the present paper results will be presented for all four cats for which measurements were made with static pressures

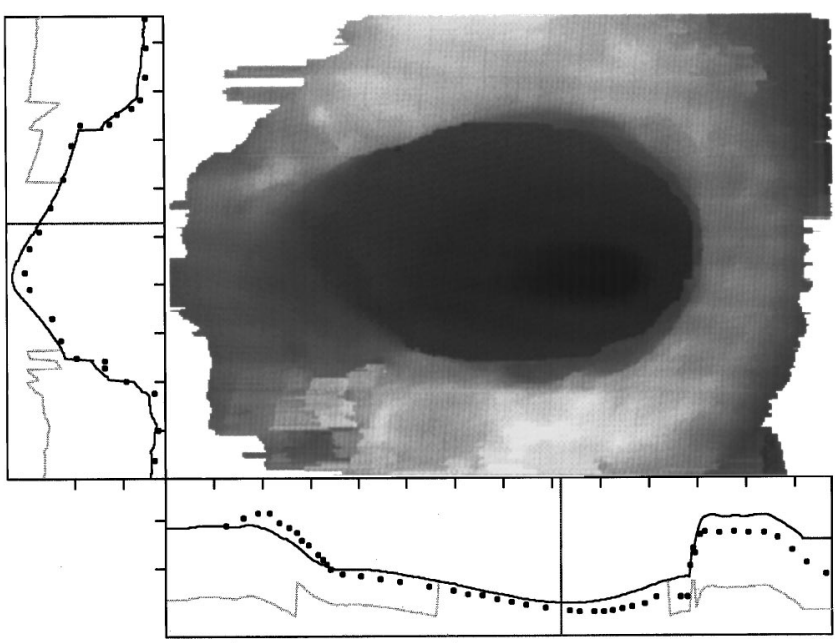

FIG. 1. Moiré image for animal CAT8, static pressure $=0$, with two profiles through the moire data and superimposed mechanically measured points. The gray levels in the moire vary between black (for the smallest $z$ values) and white (for the largest $z$ values). The profile at the left is along a line perpendicular to the manubrium, in the region of the umbo (as indicated by the vertical line in the bottom box). The profile on the bottom is along a line posterior to the manubrium (as indicated by the horizontal line in the lefthand box) and roughly parallel to it. The gray line shows the moiré profiles before phase unwrapping. The interval between tick marks is $1 \mathrm{~mm}$.

applied. Some data from one animal (CAT8) have been presented previously (Dirckx and Decraemer, 1991; Funnell et al., 1993). The moiré data for the other three animals (CTM2, CTM3, and CTM4) have not previously been published (Stoffels, 1993). For animals CAT8 and CTM2, moiré images were obtained for 9 and 11 different pressures, respectively, including one each at zero pressure and several for negative and positive pressures. For each of animals CTM3 and CTM4, two sets of pressure measurements were obtained, with each set starting at zero, increasing in several steps to a maximum, jumping back to zero, then decreasing in several steps to a minimum. For animal CTM3 there is then a third measurement at zero pressure at the end of each set. There was a time interval of about $2 \mathrm{~h}$ from the beginning of one such set to the beginning of the next. Several minutes are required to acquire the data at each pressure step.

\section{DATA-ANALYSIS METHODS}

\section{A. Phase unwrapping}

The phase-shift moiré technique results in an image matrix in which the column and row numbers of each pixel are proportional to the $x$ and $y$ coordinates, respectively, of a point on the structure being observed, and the value of the pixel is proportional to the $z$ coordinate of the point. The pixel values are actually calculated as phase angles; they are therefore subject to a $2 \pi$ ambiguity and must be "unwrapped." Because there are true abrupt jumps in the $z$ coordinates, due mostly to the overhang at the edge of the eardrum but sometimes to large displacements, conventional phase-unwrapping algorithms cannot be relied upon, and it is often difficult to unambiguously determine how to unwrap the data even visually. Figure 1 shows a profile through one particular set of data. A limited number of point-by-point 
mechanical position measurements were made in this particular animal. In spite of their relative inaccuracy these measurements do help to resolve the $2 \pi$ ambiguities, but they are extremely time consuming to make and are not usually performed.

The phase unwrapping has been carried out with a combination of manual and semiautomatic methods. A special interactive programme, MUM (Modeling Using Moiré), has been written for this purpose. The methods used include (1) automatic unwrapping of the column in the center of the image, working upward and downward from its center, followed by unwrapping of each row working leftward and rightward from the center; (2) addition or subtraction of $2 \pi$ to a single manually specified pixel, or to all pixels above, below, to the left of or to the right of such a pixel; (3) automatic addition of multiples of $2 \pi$ to all pixels in a row to minimize the differences between it and some other, previously unwrapped row; and (4) automatic image unwrapping by pairwise matching, by addition of multiples of $2 \pi$ to all pixels in an image to minimize the differences between it and some other, previously unwrapped image.

When the last method is applied to a set of images corresponding to a range of static pressures, rather than matching all of the images to be unwrapped to a single previously unwrapped image, the images are first sorted in order of their pressures and then automatically unwrapped by comparison with one another in consecutive pairs, starting with a previously unwrapped image and working upward and downward through the range of pressures. For example, if images had been measured at pressures of $-20,0,20,-40$, and $40 \mathrm{~mm}$ $\mathrm{H}_{2} \mathrm{O}$, and the image for $p=0$ had been unwrapped first, then the other images would be unwrapped by pairwise matching in the order $(0,-20),(-20,-40),(0,20),(20,40)$. This is done because the $z$-coordinate differences between two images with different pressures may well be greater than $2 \pi$ unless the pressures are close together.

\section{B. Determination of boundaries}

Once a set of images has been unwrapped, it is necessary to determine the boundaries of the pars tensa, pars flaccida, and manubrium. In some regions this is quite straightforward, but in others it is not. Figure 2 shows a set of profiles, for pressures of 220,0 , and $-220 \mathrm{~mm} \mathrm{H}_{2} \mathrm{O}$, along a line roughly perpendicular to the manubrium and running through a point approximately halfway between the short process and the umbo. Anteriorly all of the profiles converge to a point (indicated by an open circle) clearly corresponding to the edge of the eardrum. Posteriorly, however, a large jump occurs before the different profiles converge to a single $z$ value. This is because the eardrum passes underneath the overhanging ear-canal tissue, so the actual edge cannot be seen. In such cases the position of the edge was determined by visually extrapolating the profiles. (Many different pressurized profiles are used, but only two are shown in the figure for clarity.) An open circle is shown at the boundary position so estimated. The radius of the circle is $0.1 \mathrm{~mm}$, an overestimate of the possible error in the boundary position.

A determination of the boundary of the manubrium can generally be made based on the angle with which the ear-

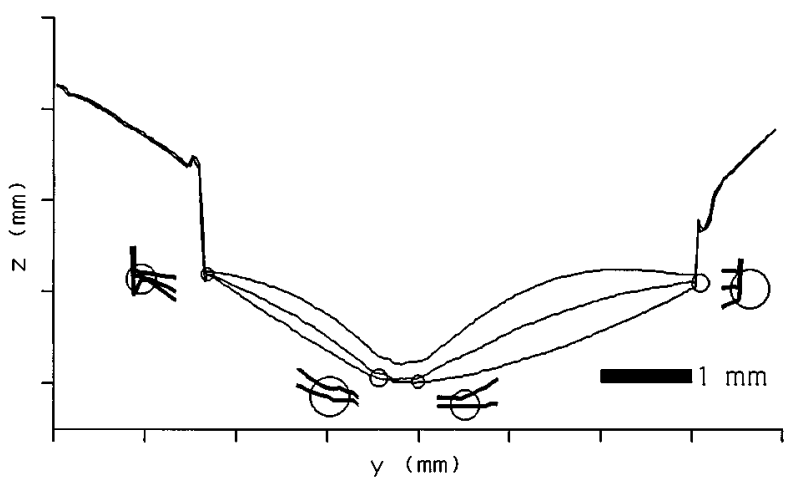

FIG. 2. Profiles through moiré data for animal CTM4, for pressures -220 (bottom line), 0 (middle), and 220 (top) $\mathrm{mm} \mathrm{H}_{2} \mathrm{O}$, along a line perpendicular to the manubrium, approximately midway between the lateral process and the umbo. The interval between tick marks is $1 \mathrm{~mm}$. The circles indicate the estimated locations of the boundaries of the pars tensa and manubrium; each circle is also shown magnified, together with the curves in its neighborhood. The sizes of the circles overestimate the possible errors of the estimates. Note that the posterior boundary of the eardrum (on the right) is beneath the overhanging ear-canal tissue.

drum meets it, and on the invariance of the cross-sectional shape of the manubrium as different static pressures are applied. In Fig. 2 the posterior edge of the manubrium is quite clear and is indicated by a small open circle. The anterior edge is somewhat less clear, and its estimated position is indicated by an open circle of radius $0.1 \mathrm{~mm}$.

In almost all cases the $x, y$, and $z$ coordinates of the edges of the pars tensa and manubrium can be estimated with confidence to within $0.1 \mathrm{~mm}$, and usually to within $0.05 \mathrm{~mm}$ or less. In many cases the superior edge of the pars flaccida is more difficult to identify, but this is assumed to be relatively unimportant for the purposes of modeling the mechanical behavior of the eardrum.

It is not possible to determine the exact boundary between the pars flaccida and the pars tensa based on the moiré data. There is often a displacement minimum in the profiles in that region, and in the present work the position of the minimum has been taken to indicate the position of the boundary. As will be seen below, however, the position in which decreased displacements are predicted by the finiteelement model does not generally correspond to the position of the boundary between the pars flaccida and pars tensa, but rather is determined by the local geometry of the model.

\section{Finite-element model generation}

\section{Definition of generic model}

The generation of finite-element models for one set of moiré data is based on a hierarchical specification of a generic model, based on points, lines, and regions. Names and interrelationships are specified for boundary lines, including the outer boundary of the pars tensa; that of the pars flaccida; the anterior and posterior boundaries between the pars tensa and pars flaccida; the manubrium; and the short process. Each line definition contains a set of six boundary conditions, corresponding to $x, y$, and $z$ displacements and to rotations about the $x, y$, and $z$ axes. These boundary conditions are used for each node to be generated along that line for the finite-element mesh. For the models discussed here, the six 
boundary conditions are either all unconstrained or all fully clamped. Each line definition may include the $x, y$, and $z$ coordinates of points that have been interactively defined for that line.

Different regions of the model are defined by concatenating directed named lines. For example, the pars tensa is defined as the region delimited by moving forward around the pars tensa outline, backward along the posterior boundary between the pars tensa and pars flaccida, backward around the manubrium, and forward along the anterior boundary between the pars tensa and the pars flaccida. For each such region are also specified (1) a set of six boundary conditions which will apply to finite-element nodes that will be generated for the interior of that region; (2) what material type the region is made of; (3) the thickness of the constituent triangular elements; and optionally (4) an element-load multiplier which by default is one but which may be set to zero for regions (like the head of the malleus) behind the eardrum and therefore not exposed to the sound pressure in the ear canal. A region definition may also contain parameters controlling the generation of a parametrically defined 3-D shape using radii of curvature as described below.

\section{Generation of finite-element mesh}

Once the coordinates of the boundary nodes have been interactively determined as discussed above, the model definition is written by the MUM programme into another file which contains the same information except that the point coordinates are associated with named points, and the line definitions are converted to explicit sequences of named points. This file is used by a hierarchical mesh generator programme (FUD) to create a finite-element model. The resolution of the finite-element mesh is specified as a nominal number of elements/diameter (Funnell, 1983).

The finite-element model is then read back in by MUM, and the new internal nodes generated by FUD are assigned $z$ coordinates by using the appropriate pixel values from a moiré image corresponding to a static pressure of zero. Occasionally one of the nodes of the finite-element mesh will be located in the part of the image corresponding to the overhang of the ear canal. In such cases the $z$ coordinate must be set manually by visual extrapolation of the profiles.

Once the $z$ coordinates have been extracted from the moiré image, each finite-element model is rotated in three dimensions so as to make the tympanic ring roughly parallel to the $x-y$ plane, to facilitate comparisons among different models.

In previous finite-element models of the cat eardrum, the three-dimensional curved conical shape was generated using a normalized radius-of-curvature parameter (Funnell, 1983). For each internal node created by the 2-D mesh generator, a $z$ coordinate was computed so that the node would lie on a circular arc whose plane lay perpendicular to the plane of the tympanic ring and whose two ends lay on the tympanic ring and on the manubrium, respectively. The radius of curvature of the arc was taken to be the product of the chord length of the arc and a normalized radius parameter. In the present work the same approach has been used to produce a parametrically defined shape for comparison with the moiré- derived shapes. The method has been enhanced so that (1) the center of each arc is taken to lie below the tympanic ring if the manubrial end is above it, but above the ring if the manubrial end is below it, so as to produce more realistic shapes near the mallear lateral process; and (2) the $z$ coordinate computed from each arc is not permitted to lie on the side of the plane of the tympanic ring opposite that of the center of curvature, which is a concern only for quite small radii of curvature. Furthermore, the normalized radius-ofcurvature parameter, rather than necessarily being a constant, is now permitted to vary either linearly or quadratically as a function of the distance around the tympanic ring of the outer end of the circular arc. It will be seen below that such a variation is required to match the behavior of models using the moiré-derived shapes.

Previous finite-element models for the cat included a fixed axis of ossicular rotation which lay in the plane of the tympanic ring (Funnell and Laszlo, 1978, etc.). In order to represent the axis more realistically when using moiré data to define the shapes (Funnell et al., 1993), the position of the axis was established based on the approximate positions of the posterior incudal ligament and anterior mallear process in a 3-D reconstruction of the cat middle ear (Funnell and Phelan, 1981; Funnell and Funnell, 1989). Because of the additional complexity that this introduces to a comparison of different individual animals, in the present work the issue of the position and nature of the axis of rotation is sidestepped by simulating the condition of a fixed manubrium. This is directly comparable to a feasible experimental situation and permits concentration on the characteristics of the eardrum and its shape.

In all of our previous finite-element models of the cat eardrum the boundary between the pars flaccida and pars tensa was modeled as a thickened "ligament." No such thickening was reported by Lim (1968), however, and examination of serial sections has indicated that none occurs in the cat. In fact, it seems to be hardly possible to distinguish sharply between the pars flaccida and the pars tensa with light microscopy in histological sections: The characteristic lack of a lamina propria in the pars flaccida (Lim, 1968) is not evident at low magnifications, and the change in thickness is gradual. In the present models the boundary has been taken, for lack of a better choice, to coincide with the region of smaller static displacements between the larger ones seen in the pars flaccida and pars tensa, respectively.

In previous models the thickness of the pars flaccida was unimportant because no internal nodes were generated for it. For the present models we have included internal nodes, and have taken the thickness of the pars flaccida to be $80 \mu \mathrm{m}$. For the Young's modulus being used $\left(10^{7} \mathrm{dyn} \mathrm{cm}^{-2}\right)$ this results in more or less reasonable pars-flaccida displacements. The characteristics of the pars flaccida have little effect on the mechanical behavior of the rest of the eardrum.

Except as discussed above, the model parameters used for the calculations presented here are the same as those used in our previous models. Inertial and damping effects are ignored, so the results are applicable for low frequencies, up to at least $300 \mathrm{~Hz}$. The thickness, Young's modulus and Poisson's ratio of the pars tensa are $40 \mu \mathrm{m}, 2 \times 10^{8} \mathrm{dyn}^{-2}$ and 


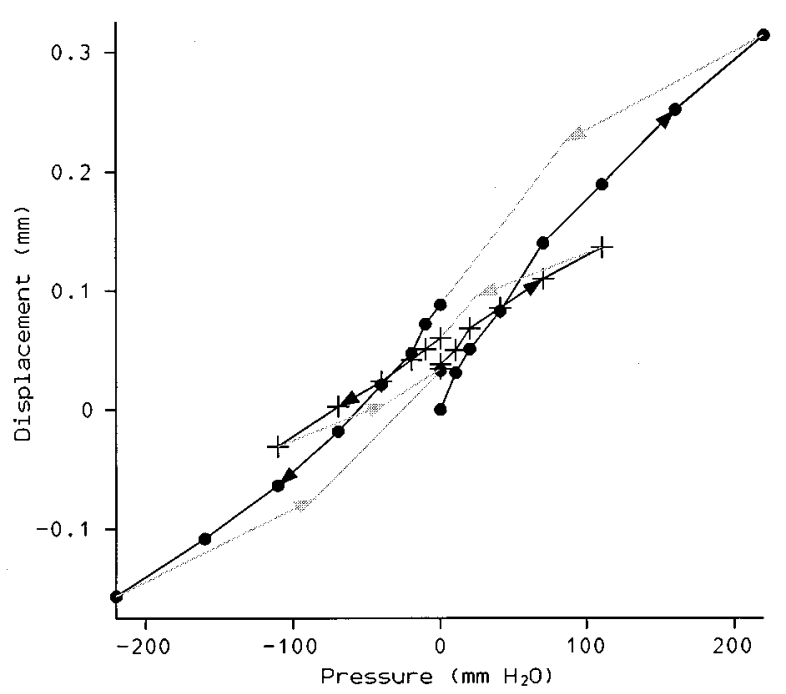

FIG. 3. Pressure-displacement curves for a location in the posterior pars tensa of animal CTM3. The zero reference for the vertical scale is arbitrary. The arrows indicate the temporal order of the measurements. The circles (-) correspond to the first cycle of pressures and the crosses $(+)$ correspond to the second. The gray lines are intended to suggest the forms of the hysteresis loops and do not correspond to actual measurements.

0.3 , respectively. The stimulus is a uniform pressure of 100 dB SPL.

As always in finite-element modeling, the fineness of the mesh must be chosen as a compromise between decreased accuracy and increased computational expense. For one of the eardrums considered here, models were generated with nominal mesh resolutions of 20, 30, and 40 elements/ diameter. The maximal eardrum displacements were computed to be 518.6, 526.4, and $526.7 \mathrm{~nm}$, respectively, and the overall displacement patterns were very similar for 30 and 40 elements/diameter. This indicates that 30 is an adequate resolution and that is the value used for the following.

\section{MEASURED SHAPES}

Since the focus of this paper is on the resting $(p=0)$ shapes, and since the measurements with static pressures applied were intended primarily to aid in determining boundaries for the models, no detailed presentation will be made of the displaced shapes. It is important, however, to take a brief look at the eardrum displacements as a function of pressure in order to understand the variations in the resting shapes within individual animals. Figure 3 shows, for the animal (CTM3) in which the pressure variations were measured most systematically, pressure-displacement curves for a single point in the posterior region of the pars tensa, roughly midway between the tympanic ring and the manubrium. Positive displacements are outward into the ear canal, and correspond to a more highly curved eardrum surface.

Note that the pressure-displacement curves exhibit hysteresis, that is, the eardrum does not return to the same position when the pressure returns to zero from a positive excursion as when the pressure returns to zero from a negative excursion. This is a common feature of the behavior of biological materials (Fung, 1993). It is reflected in the wellknown fact that impedance tympanometry measurements

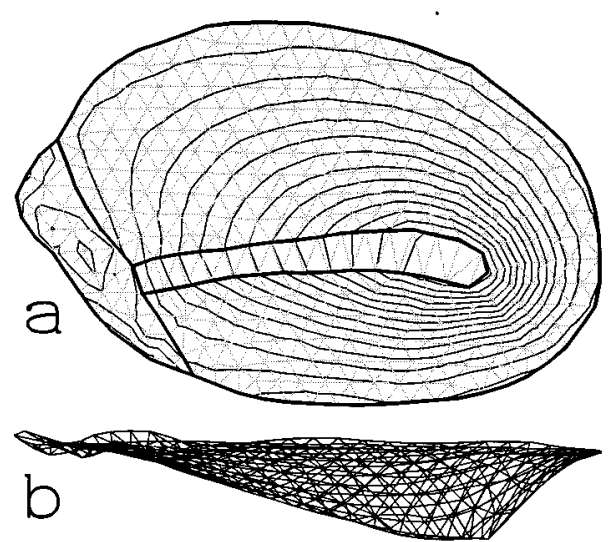

FIG. 4. Shape of finite-element model for animal CTM4. (a) Contour lines of constant $z$, with an interval between contours of $0.1 \mathrm{~mm}$. The gray lines show the triangles of the finite-element mesh. (b) Side view of finiteelement model.

give different results depending on the order in which the pressure is varied (Osguthorpe and Lam, 1981), and has also been reported in moire measurements in the gerbil (von Unge et al., 1993). The behavior of such a material depends in general upon its history, and the shape of the hysteretic force-displacement loop may change from one cycle to the next. If the material is taken through the same load cycle repeatedly, the force-displacement curve will normally converge to a stable shape; the material is said to have been "preconditioned." The fact that the second loop in Fig. 3 exhibits much smaller displacements than the first loop may indicate that more preconditioning cycles were required, or it may reflect gradual post-mortem changes. Similar behavior was found for the other animal (CTM4) in which two pressure cycles were measured.

One of the resting $(p=0)$ eardrum shapes for animal CTM4 is represented in Fig. 4 by means of (a) the $x-y$ projections of the finite-element model with superimposed constant $-z$ contours; and (b) the $x-z$ projection of the model. The contour lines appear relatively evenly spaced, except near the tympanic ring where they are further apart. This indicates a small curvature in the inner part of the eardrum. The same thing can be seen in the $x-z$ view: The profile between the umbo and the tympanic ring is almost a straight line except near the ring.

The various resting shapes measured for all four animals are summarized in Fig. 5 as constant $z$ contours. The different shapes measured for a given animal are visually very similar, but there are visible differences among animals. In animals CAT8, CTM2, and CTM3, the spacing between contours tends to increase gradually from the manubrium outward, rather than remaining more or less constant until near the tympanic ring as it does in animal CTM4. The eardrums of all four animals are approximately the same diameter and approximately the same depth.

\section{SIMULATION RESULTS}

Figure 6 shows low-frequency displacement-amplitude contours of the finite-element model generated for animal CTM4, corresponding to the mesh shown in Fig. 4. The 

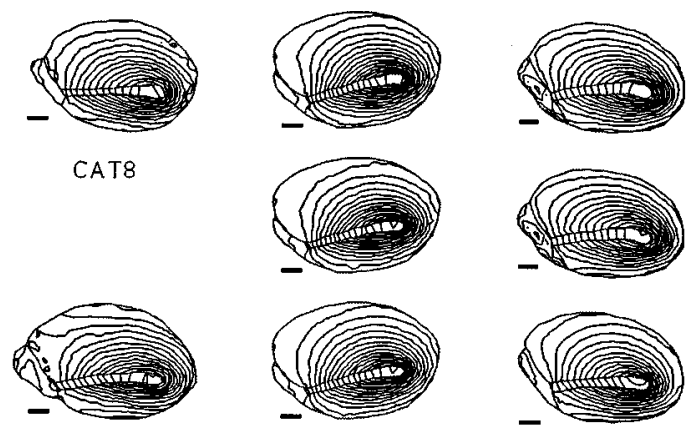

CTM2
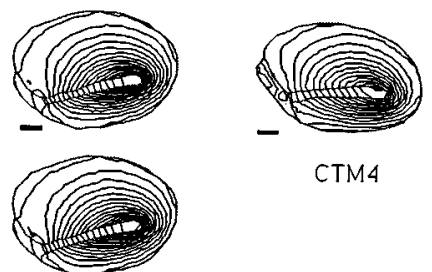

CTM4

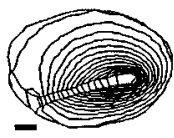

CTM3
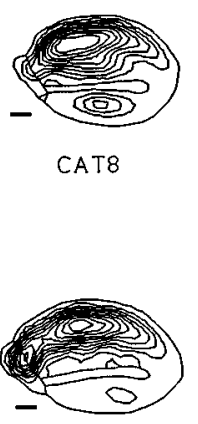

CTM2
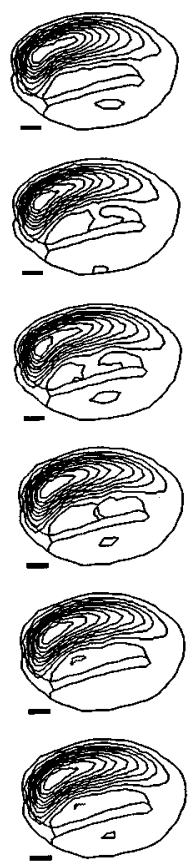

CTM3
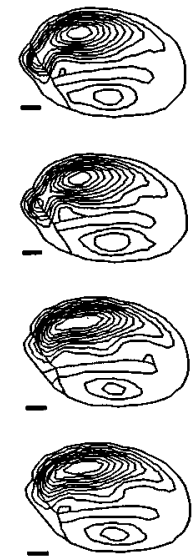

CTM4
FIG. 5. Shapes of finite-element models for all animals, as in Fig. 4(a). There is one model each for animals CAT8 and CTM2; there are six models for CTM3 and four for CTM4, corresponding to different resting shapes. Since the models differ slightly in size, a separate 1-mm scale bar is shown for each. As in Fig. 4, the $\Delta z$ between contour lines is $0.1 \mathrm{~mm}$.

maximal displacement on the eardrum is $526 \mathrm{~nm}$ and occurs in the posterior part of the pars tensa. In the anterior region of the pars tensa there is a secondary maximum approximately one quarter as large. Figure 7 shows similar displacement contours corresponding to all of the models shown in Fig. 5. Just as for the shapes themselves, the simulated vibration patterns are similar to each other within animals but are significantly different in different animals. The maximal displacements for animal CTM4 range from 526 to $620 \mathrm{~nm}$, and the anterior maximum is always $20 \%$ to $25 \%$ as large as the posterior maximum. In animal CTM3 the posterior maximum is located more superiorly than in CTM4, and the anterior displacement maximum is only about $10 \%$ as large as the posterior one. The displacements just posterior to the manubrium are also very small, and are actually slightly negative in some cases. In animal CAT8 the posterior displacements are small, with a maximum of only $487 \mathrm{~nm}$, but the anterior displacements are relatively large (up to about

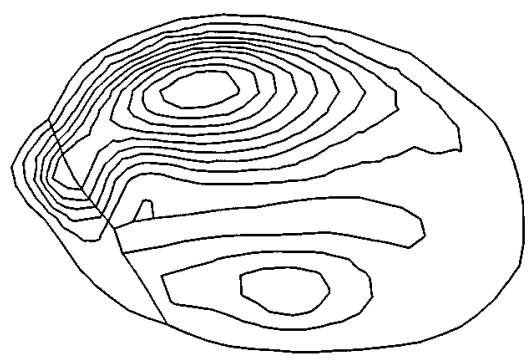

FIG. 6. Simulated displacements for the model shown in Fig. 4. The contours are lines of constant displacement amplitude, evenly spaced between 0 and the maximum displacement.
FIG. 7. Displacements for the models shown in Fig. 5, displayed as in Fig. 6. The maximum displacements are $487 \mathrm{~nm}$ for CAT8; $782 \mathrm{~nm}$ for CTM2; $528,617,603,574,619$, and $614 \mathrm{~nm}$, respectively, for the six CTM3 cases; and 526, 559, 591, and 620, respectively, for the four CTM4 cases.

$30 \%$ of the posterior maximum). In animal CTM2 the posterior displacements are large (maximum $=782 \mathrm{~nm}$ ) but the anterior displacements are relatively small.

Among the four resting shapes measured for animal CTM4, the first resulted in the smallest simulated maximal displacement. For a model based on the first shape, decreasing the Young's modulus of the pars tensa by about $15 \%$ increases the maximal displacement to the largest value found for that animal. Decreasing the Young's modulus in the same model by about $33 \%$ increases the maximal displacement to the largest value found among all four animals, while increasing it by about $8 \%$ decreases the maximal displacement to the smallest value found among all four animals. Similarly, decreasing the thickness of the pars tensa by about $22 \%$, or increasing it by about $5 \%$, changes the maximal displacement so it equals the largest or the smallest values, respectively, among all four animals. Unlike changes in the eardrum shape, changes in either the Young's modulus or the thickness have very little effect on the overall shapes of the simulated vibration patterns.

In animal CTM2 there is a displacement maximum near the putative boundary between the pars flaccida and pars tensa which is almost as large as the displacement maximum of the pars tensa proper. In the first two models for CTM4 there is also a maximum near that boundary but it is smaller. In the other models there is no displacement maximum in the neighborhood of the pars flaccida. Recall that the boundary between the pars flaccida and pars tensa was assumed to lie at the location of the reduced displacements that occur between the pars-flaccida maximum and the pars-tensa maximum in the response to large static pressures. The simulation results clearly do not support such an approach, but there is 
no other way of determining the boundary based on the moiré data.

There is a noteworthy pattern within the simulation results for the animals (CTM3 and CTM4) for which more than one resting shape was measured: Within each cycle of pressures from zero to positive to zero to negative, the model corresponding to the shape after the positive excursion produces a greater maximal eardrum displacement than the model corresponding to the shape before the positive excursion. The increase was $17 \%$ and $8 \%$ for the two pressure cycles for animal CTM3, and 6\% and 5\% for CTM4. For animal CTM3, for which a third resting shape was measured after the negative pressure excursion, the simulated displacement for the shape after the negative excursion was slightly less $(2 \%$ and $1 \%)$ than that for the shape before the excursion. These results are somewhat surprising since (except for the last-mentioned, $1 \%$ change) the increased displacements correspond to eardrum shapes which are more highly curved. A more highly curved structure is usually stiffer, and displaces less, than a similar structure which is less curved. A more detailed examination of the different configurations of the eardrum and manubrium would be required to try to explain the apparent discrepancies. Experiments with the manubrium fixed would be useful in removing one confounding factor.

An evaluation of the sensitivity of the models to errors in determining the exact coordinates of the eardrum boundary was performed (Funnell et al., 1993) by generating several models (with mobile manubrium) for the CAT8 data with pars-tensa and pars-flaccida boundaries that were expanded or contracted by various amounts. Over a range of expansion/contraction of $\pm 5 \%$, which corresponds to a boundary shift of approximately $0.12 \mathrm{~mm}$ (for the shorter diameter of the eardrum) to $0.2 \mathrm{~mm}$ (for the longer diameter) and is more than the likely range of inaccuracy in our boundary estimates, the maximal eardrum displacement changed by $+10 /-14 \%$. Several models were also generated by shifting the pars-tensa and pars-flaccida boundaries in the $z$ direction by varying amounts. Shifts of $\pm 0.1 \mathrm{~mm}$ caused the maximal eardrum displacement to change by $+4 /-15 \%$. These numbers suggest that $15 \%$ is a reasonable upper limit for errors in simulated eardrum displacements due to errors in boundary determination. The manubrial displacement varied much more $(+51 /-41 \%$ for boundary expansion and $+40 /-39 \%$ for vertical shifts), probably because of the short distance between the tip of the manubrium and the tympanic ring, but this is not relevant for the present paper since a fixed-manubrium condition is being modeled here.

In our previous models, the curvature of the sides of the eardrum was modeled by a single normalized radius-ofcurvature parameter, as discussed above. For comparison, several models (with mobile manubrium) were generated that way using the boundaries obtained from the moire data for animal CAT8. Simulations with a range of radii showed that no such model could reproduce the behavior of the real shape, in the sense of producing reasonable displacements for both the eardrum and the manubrium in the same model (Funnell et al., 1993).

Similarly, models (with fixed manubrium) have been generated for animal CTM4 using normalized radii of curvature ranging from 1.0 to 15 . A model with a parameter of 15 produced the correct magnitude of displacements in the posterior region of the pars tensa but the anterior displacements were about twice as large as with the moiré-derived shape. Conversely, a parameter of 2.5 produces acceptable anterior displacements but the posterior displacements are about $40 \%$ too small.

With a normalized radius-of-curvature parameter which varies linearly from 15 posterosuperiorly to 1.0 anterosuperiorly, the model produces maximal posterior and anterior displacements which match those produced by the model with moiré-derived shape. The shapes of the displacement patterns do not, however, match those for the moiré-derived shape so closely, and some other method would be required to adequately parametrize the shape of the eardrum.

\section{CONCLUSIONS}

The phase-shift moiré technique, combined with the analysis methods outlined here, makes it feasible to obtain detailed shape data from experimental animals and to incorporate the data in models of individual animals. The behavior of the model, especially the displacement of the manubrium, is quite sensitive to errors in the position of the eardrum boundary. The application of static pressures to the eardrum facilitates the definition of the boundary. The ear canal can, with care, be resected to within about $0.5 \mathrm{~mm}$ of the eardrum without damaging the latter, but even so parts of the boundary of the eardrum will be hidden behind overhanging ear-canal tissue. A visual extrapolation of the displaced curves permits estimation of the position of the boundary to within less than $0.1 \mathrm{~mm}$. The simulation results suggest that this error in boundary position would not cause an error in maximum predicted eardrum displacement of more than $15 \%$.

A significant amount of effort is required to unwrap the $z$-coordinate measurements, which are computed as phases and are subject to $2 \pi$ ambiguities. Software has been written which implements a number of automatic, semiautomatic, and manual unwrapping techniques. One technique which is being investigated to facilitate the process of unwrapping is the use of two gratings with different line spacings. For example, a grating with 3 lines/mm could be used to supplement the 4-line/mm grating currently used. The $2 \pi$ ambiguities for the two gratings would correspond to different $\Delta z$ values, which would help to resolve ambiguities definitively.

The results presented here quantify the observation that the shape of the eardrum, and hence its mechanical behavior, depends upon its displacement history. This fact implies that care must be taken to properly precondition the eardrum in order to obtain repeatable measurements, whether of point displacements or of volume displacements. More work will be required to determine what preconditioning procedures are adequate for different experimental situations.

Although all four eardrums considered here are approximately the same size and depth, there are significant differences in the detailed shapes and curvatures, and also in the displacements predicted by the corresponding finite-element 
models. The differences within animals are smaller in spite of the effects of the eardrum's stress-strain history on its resting shape.

The simulation results suggest that the experimentally observed decreases in displacement in the neighborhood of the boundary between the pars tensa and pars flaccida do not in fact correspond to the position of that boundary, and are determined by the local geometry and curvatures rather than by the structural properties of the boundary.

The method previously used to parametrically summarize the shape of the eardrum, involving a single radius-ofcurvature parameter, has been shown to be inadequate to represent the true shape of the eardrum. Use of a variable radius-of-curvature parameter improves the representation of the shape but further work will be required to evaluate its accuracy and usefulness.

\section{ACKNOWLEDGMENTS}

Work supported by the Medical Research Council of Canada and the University of Antwerp. We thank J. Lauzière for editing the manuscript.

\section{DEDICATION} 1995.

This paper is dedicated to H. John Funnell, CD, 1922-

Decraemer, W. F., and Dirckx, J. J. J. (1991). “Moiré-shift interferometer measurements of the shape of human and cat tympanic membrane," in Holography, Interferometry and Optical Pattern Recognition in Biomedecine, SPIE Proceedings of Biomedical Optics '91, edited by H. Podbielska (Soc. Photo-Opt. Instrum. Eng., Bellingham, WA), pp. 26-33.

Decraemer, W. F., Dirckx, J. J. J., and Funnell, W. R. J. (1991). "Shape and derived geometrical parameters of the adult, human tympanic membrane measured with a phase-shift moiré interferometer,' Hear. Res. 51, 107122.

Dirckx, J. J. J., and Decraemer, W. F. (1989). 'Phase shift moiré apparatus for automatic 3-D surface measurement,' Rev. Sci. Instrum. 60, 36983701 .

Dirckx, J. J. J., and Decraemer, W. F. (1990). "Automatic calibration method for phase shift shadow moiré interferometry," Appl. Opt. 29, 1474-1476.

Dirckx, J. J. J., and Decraemer, W. F. (1991). "Human tympanic membrane deformation under static pressure," Hear. Res. 51, 93-105.
Dirckx, J. J. J., and Decraemer, W. F. (1992). "Area change and volume displacement of the human tympanic membrane under static pressure," Hear. Res. 62, 99-104.

Dirckx, J. J. J., Decraemer, W. F., and Dielis, G. (1988). "Phase shift method based on object translation for full field automatic 3-D surface reconstruction from moiré topograms,"' Appl. Opt. 27, 1164-1169.

Dirckx, J. J. J., Decraemer, W. F., and Janssens, J. L. (1986). "Real-time shadow moiré vibration measurement: method featuring simple setup, high sensitivity, and exact calibration,'” Appl. Opt. 25, 3785-3787.

Fung, Y. C. (1993). Biomechanics: Mechanical Properties of Living Tissues (Springer-Verlag, New York), 2nd ed., xviii +568 pp.

Funnell, W. R. J. (1981). "Image processing applied to the interactive analysis of interferometric fringes,' Appl. Opt. 20, 3245-3250.

Funnell, W. R. J. (1983). "On the undamped natural frequencies and mode shapes of a finite-element model of the cat eardrum,' J. Acoust. Soc. Am. 73, 1657-1661.

Funnell, W. R. J., Decraemer, W. F., Dirckx, J. J. J., and Khanna, S. M. (1993). "On the effects of shape variations in a finite-element model of the cat eardrum," 16th ARO Midwinter Meeting.

Funnell, S. M., and Funnell, W. R. J. (1989). "An approach to finiteelement modeling of the middle ear," J. Acoust. Soc. Am. Suppl. 185 , S67.

Funnell, W. R. J., and Laszlo, C. A. (1978). "Modeling of the cat eardrum as a thin shell using the finite-element method,' J. Acoust. Soc. Am. 63, 1461-1467.

Funnell, W. R. J., and Phelan, K. E. (1981). "Finite-element modeling of the middle-ear ossicles,'” J. Acoust. Soc. Am. Suppl. 1 69, S14.

Helmholtz, H. L. F. (1869). "The mechanism of the middle-ear ossicles and of the eardrum,' Pflüg. Arch. ges. Physiol. 1, 1-60 (in German).

Khanna, S. M., and Tonndorf, J. (1975a). "Tympanic membrane shape determined by moiré topography,', J. Acoust. Soc. Am. Suppl. 1 57, S72.

Khanna, S. M., and Tonndorf, J. (1975b). "Tympanic membrane: effect of dc pressure changes in the middle ear of cats,' J. Acoust. Soc. Am. Suppl. 1 58, S88.

Kirikae, I. (1960). The Structure and Function of the Middle Ear (Univ. Tokyo, Tokyo), vi+157 pp.

Kojo, Y. (1954). "Morphological studies of the human tympanic membrane,’ J. O.-R.-L. Soc. Jpn. 57, 115-126 (in Japanese).

Lim, D. J. (1968). "Tympanic membrane Part II: Pars flaccida," Acta Otolaryngol. 66, 515-532.

Osguthorpe, J. D., and Lam, C. (1981). "Methodologic aspects of tympanometry in cats," Otolaryngol. Head Neck Surg. 89, 1037-1040.

Stoffels, E. J. A. J. (1993). "Geautomatiseerde moiré topografie, en zijn toepassing voor vorm-, vervormings -en diktemetingen aan het kattetrommelvlies,' unpublished report of a period of practical training, Hogeschool Eindhoven, Faculty of Technics, Technical Physics (in Dutch).

von Unge, M., Decraemer, W. F., Bagger-Sjöbäck, D., and Dirckx, J. J. J. (1993). "Displacement of the gerbil tympanic membrane under static pressure variations measured with a real-time differential moiré interferometer,' Hear. Res. 70, 229-242. 